\title{
Compensatory Paraoxonase and arylesterase levels in Hyperbaric Oxygen Treatment OF Sudden Sensorineural Hearing Loss
}

\author{
Esin Eren ${ }^{1)}$, Necat Yilmaz ${ }^{1)}$, Furkan Yildirim²), Ozlem Giray3) \\ 1) SBÜ Antalya education and Research Hospital Central Toxicology Laboratories \\ 2) SBÜ Antalya education and Research Hospital Underwater and Hyperbaric Medicne \\ 3) SBÜ Antalya education and Research Hospital Pharmacology
}

\begin{abstract}
Objective: Paraoxonase1 (PON1) and Arylesterase (ARE) levels are associated with reduced risk of atherosclerosis. The functional status of high density lipoprotein (HDL) is closely related to the PON1/ARE enzyme activity. Functional changes in treatment of sudden sensorineural hearing loss (SSNHL) may be achieved by post-translational modification of lipid metabolism induced by hyperbaric oxygen therapy (HBOT).

Methods: Men patients with SSNHL who met the research criteria were included in the study. HBOT was performed on average 30 sessions. Laboratory measurements were made at the beginning and end of HBOT for the same patients. Serum levels of PON1/ARE and routine lipid laboratory parameters were measured to determine possible changes in SSNHL after HBOT.

Results: In this study, a reducing effect on PON1 enzyme amount of long-term HBOT was detected. The serum PON1 amount of patients with SSNHL was $19.7 \pm 2.7 \mathrm{ng} / \mathrm{mL}$ (mean $\pm \mathrm{SD}$ ) before HBOT, and the serum PON1 decreased to $17.0 \pm 2.1 \mathrm{ng} / \mathrm{mL}$ (mean \pm SD) after 30 sessions of HBOT. This decrease in PON1 levels was statistically significant $(p=0.035)$. There was also a statistically significant decrease in the enzyme activity of ARE in the SSNHL patients $(p=0.024)$.

Conclusion: This preliminary study showed a significant decrease in serum PON1/ARE enzyme content in SSNLH patients with HBOT. In fact, it can be assumed that HBOT has no adverse effect on HDL functionality. However, the decrease in PON1 level by HBOT with 30 or more sessions may be important for the antioxidant function of HDL.It may possibly cause post-translational changes in antioxidant defense mechanisms due to increased oxidative stress with HBOT. In conclusion, larger clinical studies are needed to determine the possible effects of HBOT on HDL-related PON1/ARE functionality in SSNHL.

Keywords: Paraoxonase; Hyperbaric oxygen therapy; Inflammation; Oxidative stress; Reactive oxygen species Arylesterase.
\end{abstract}

\section{ARTICLE INFO}

PolHypRes 2020 Vol. 70 Issue 1 pp. $47-52$

ISSN: $1734-7009$ elSSN: $2084-0535$

DOI: $10.2478 /$ phr-2020-0004

Pages: 6, figures: 1, tables: 2

page www of the periodical: www.phr.net.pl

Original article

Submission date: 17.01.2020 r.

Acceptance for print: 14.02.2020 r.

Publisher

Polish Hyperbaric Medicine and Technology Society 


\section{INTRODUCTION}

HBOT acts as a primary or secondary treatment for a variety of medical conditions. The use of HBOT has increased markedly in various diseases over the past decade [1]. The etiology for sudden sensorineural hearing loss (SSNHL) is defined in only $10 \%$ of cases, whereas the rest are labeled as idiopathic [2-4]. So, how can be HBOT effective with a common molecular pathway in treatment of SSNHL? SSNHL was first described in 1944 and its incidence is $5-20 / 100000$. [4]. However, the incidence is thought to be higher due to the fact that patients who recover spontaneously do not seek medical attention. The reason for the etiology of sudden hearing loss is not found in many patients and is considered idiopathic [5]. Little is known about the association between SSNHL and oxidative stress [3]. In addition, endothelial dysfunction and vascular diseases are also involved in etiopathogenesis of SSNHL [6]. In particular, the feeding of the cochlea from the posterior cerebral circulation with a single terminal branch may explain the sudden and rapid course in the SSNHL of vascular etiopathogenesis. In addition, many clinical studies have shown that SSNHL patients often present with systemic arterial hypertension, diabetes mellitus and dyslipidemias with atherosclerosis, and thromboembolic risk [2-6].

Serum lipid changes associated with HBOT have not yet been well identified in humans, particularly with a focus on HDL functionality [1,7].We could not find a study showing the effect of treating SSNHL patients with HBOT on the enzymes PON1/ARE, which is linked to HDL, in literature. In previous research, the combined effect of hyperbaric oxygen pressure has led to a significant improvement in tissue oxidation. It also revealed that antiapoptotic and anti-atherosclerotic effects increased mitochondrial metabolism. It is known that elevation in free radical formation or intensive antioxidant molecule consumption after HBOT leads to post-translational modification of biological macromolecules such as lipids, lipoproteins, and enzymes [8].

However, there are no studies in the literature showing the effect of HBOT on PON1/ARE in SSNHL patients. Recently effect of decreased anti-inflammatory and antioxidant activities of PON1 with HBOT has been shown in different pathologies [1]. Naturally, PON1/ARE is an HDL-associated enzyme activity with strong antioxidative and athero-protective effects, with the potential to hydrolyze oxidized low density lipoprotein (LDL). Our aim in this study was to evaluate the possible effect of prolonged HBOT on HDL-associated PON1/ARE levels in SSNHL patients.

\section{MATERIAL AND METHOD}

\section{STUDY POPULATION AND CLINICAL EXAMINATIONS}

In this prospective cross sectional research 43 patients between the ages of 20-52 years were included in this study as a prospective work 6-month period with HBOT (mean age: $39.3 \pm 12.7$ ). For this purpose, male patients who applied to the Department of Hyperbaric Medicine with the diagnosis of sudden hearing loss were evaluated according to clinical diagnosis / follow-up. All patients received a comprehensive physical examination, a generalized questionnaire, and informed consent before being included in the study. In the survey: age, socioeconomic status, ancestral roots, physical activity status, smoking, alcohol consumption and detailed medical history were recorded. Patients were excluded if they had any of the following: hearing loss caused by rupture of round windows membrane, concomitant marked vestibular symptoms, autoimmune diseases, upper respiratory tract infection in the last 4 weeks, fluctuating hearing loss, or contraindications to our treatment protocol.

Body mass index was measured by dividing weight in kilograms by height by square meters. According to the American Heart Association / American College of Cardiology, hypertension was defined as a systolic blood pressure of at least $140 \mathrm{mmHg}$ and diastolic blood pressure of at least $90 \mathrm{mmHg}$. As a result, blood pressure was measured manually in all patients.Primary, the criteria for participation consisted of patients older than 18 who had no contraindication to HBOT. Criteria for exclusion were included smoking, alcohol consumption, existing anti-lipid and antioxidant treatments. Clinical and laboratory data were recorded for all patients. The protocol was approved by the Institutional Review Board and carried out in accordance with the revised Helsinki Declaration in 2001. Informed consent was taken from all participants.

\section{HBOT PROTOCOL}

Each successive HBOT sessions were held five days a week, with each session being 120 minutes. In a multi-atmosphere hyperbaric chamber (Baroks, MUL $35)$, each participant breathes $100 \%$ oxygen in 2.5 atmospheres, with air break times of five minutes every 30 minutes. Each HBOT session also had 15-minute compression and 10-minute decompression times.

\section{BLOOD SAMPLING AND LABORATORY MEASUREMENTS}

\section{BLOOD SAMPLE COLLECTION}

Before beginning the HBOT, blood samples were taken from all patients after 12 hours of starvation. Therefore, blood samples were taken in the mornings after a night of fasting in study. During the HBOT of SSNHL patients, 20 th and 39 th in between HBOT sessions, once again starvation blood was taken. The blood were then centrifuged for 15 minutes at 3,000 rpm and the serum was then stored at $-80{ }^{\circ} \mathrm{C}$ for PON1 and ARE enzyme analysis. Routine lipid parameters were measured immediately.

\section{ROUTINE LABORATORY PARAMETERS}

Triglycerides (TGs), total cholesterol (TC), LDLcholesterol (LDL-C), HDL-cholesterol (HDL-C) and serum creatinine levels, an auto analyzer (Architect c16000, Abbott Diagnostics) assay with commercially available kits (Abbott) was determined using. 


\section{MEASUREMENT $\quad$ OF $\quad$ PON1 CONCENTRATIONS}

The samples were tested for Human PON1 concentrations (Biont, catalog no: YLA0984HU), using a sandwich enzyme-linked immunosorbent experiment, according to the manufacturer's instructions. The PON1 concentrations results was expressed as $\mathrm{ng} / \mathrm{ml}$.

\section{MEASUREMENT OF ARE ENZYME ACTIVITY}

PON1/ARE enzyme activity were measured by using commercially available kit (Relassay®). Phenyl acetate was used as a substrate to measure serum ARE activity. The molar absorptivity of the colored complex is $400 \mathrm{~m} 2 / \mathrm{mol}$ and $1 \mathrm{U}$ of PON1/ARE activity is equal to $1 \mathrm{mmol}$ of phenyl acetate hydrolyzed per liter per minute at $37^{\circ} \mathrm{C}$. Double-distilled water $(\mathrm{ddH} 2 \mathrm{O})$ was used as a negative control during spectrometry. The activity of PON1/ARE was expressed as kU/L.

\section{STATISTICAL ANALYSIS OF DATA}

Continuous variables were presented as mean \pm $\mathrm{SD}$, while categorical variables were presented as numbers and percentages. Statistical analysis MedCalc statistical software version 15, 8 (MedCalc Software Bvba, Ostend, Belgium; https://www.medcalc.org; 2018) was made using. The Kolmogorov-Smirnov test was used to evaluate the distribution of continuous variables, the pairedsampled t-test, Pearson Chi-Square test for frequencies were used to compare measurements. P-value $<0.05$ was considered statistically significant.

\section{RESULTS}

The demographic and clinical characteristics of the subjects included in this study are shown in Table 1. The mean body mass index of the patients was $22.7 \pm 3.8$ $\mathrm{kg} / \mathrm{m} 2$ and the number of HBOT sessions received ranged from 20-39 sessions(mean: 30). No adverse side effects or unexpected clinical signs were observed in patients receiving HBOT. Treatment responders were divided into two groups; healing (full recovery) and no recovery (Table 1).

At the beginning before treatment, serum PON1 levels were $19.7 \pm 2.7 \mathrm{ng} / \mathrm{ml}$ (mean \pm SD). However, after 30 sessions, serum PON1 amount was reduced to $17.0 \pm 2.1$ $\mathrm{ng} / \mathrm{ml}$. At the end of the average 30th session, this decrease in PON1 was statistically significant $\mathrm{p}=0.0036$.

Also serum ARE enzyme activitiy were significantly lower in patients with SSNHL in after HBOT $(228.4 \pm 51.2 \mathrm{kU} / \mathrm{L})$ compared to before HBOT $(178.2 \pm$ $42.5 \mathrm{kU} / \mathrm{L}), \mathrm{p}=0.024$. In addition, mean serum lipid levels for both pre-and post-HBOT are given in Table 2. As expected there was no statistically significant difference in all routine lipid parameters after HBOT.

\section{DISCUSSION}

Firstly, this study shows that long term HBOT were also might associated with changes in HDL metabolism. Because it is known that the imbalance of free radical formation or intensive consumption of antioxidant molecules leads to relatively decreased of biological macromolecules such as serum PON1 levels [9]. A balance shifting towards increased oxidative stress in SSNHL has been recently reported [2]. The most important finding of this study is, of course, the decrease in the PON1 concentrations in the serum of patients receiving HBOT. It should be kept in mind that HDL-functionality is mostly made up of activity of PON1/ARE [1]. The importance of this study lies in measuring the response to HBOT of HDLrelated PON1/ARE enzymes sensitive to changes in oxygen concentration in SSNHL disease. This findings may be important in an environment where patients are exposed to extremely high concentrations of oxygen, such as during HBOT.

In the literature, there is very little research showing the effect of HBOT on molecules. For example, in one study, changed levels of platelet activating factor was demonstrated in diseases after HBOT. As a result, it has been reported that the platelet activating factor increases after HBOT [8]. A recent study also reported a well increase in HBOT and HDL-associated ApoA-1 levels [1]. Similarly, data obtained in this study demonstrated a significant decrease in serum PON1 levels and HDL-related antioxidant ARE enzyme activity in SSNHL patients. Whereas, TC, HDL-C, TGs, and TGs/HDL-C levels were not significantly changed. According to these results; it is possible to deal with oxidative stress that may occur as a result of high oxygen pressure applied during HBOT, but it can be considered that HDL-related antioxidant molecules are reduced due to higher consumption of PON1/ARE enzymes. The fact that we have similar findings with a study in the literature supports this idea [9].

The study, which reported the decreased amount of PON1 with HBOT in the literature, generally included a number of different types of diseases along with SSNHL $[1,10]$. However, the improvement of the majority $(88 \%)$ of SSNHL patients participating in this study showed the effectiveness of the response to HBOT. In addition, the information in the literature reported a relationship between HBOT and the dehydration of patients, which may lead to a relative change in serum parameters [11]. Although all of the patients started steroid therapy prior to HBOT, these positive changes were observed after HBOT therapy. Optimal criteria for HDL functionality and a standardized regimen for HBOT should be applied in routine practice. If 30 sessions or more of HBOT will be applied to SSNHL patients, PON / ARE enzyme activity protective applications can be added to the treatment protocol. So, this study is not without limitations. But to our knowledge, this is the first report demonstrating decreased of serum PON1, in SSNHL patients after HBOT.

\section{Conclusions}

Presumably, antioxidative responses can be increased by oxidant response. In several studies antioxidants have been used as a therapeutic measure for causes of hearing loss. The results we obtained in this study regarding HBOT should be supported by wider and long-term studies. There is no doubt that HBOT will to take a wider place in the future in modern medical use. Finally, these findings may suggest that serum HDL-related PON1 and ARE levels are important factors for response the HBOT in SSNHL patients. 
Clinical characteristics of SSNHL patients and laboratory findings.

\begin{tabular}{lll}
\hline Parameters & Mean $\mathbf{\pm}$ SD & Range \\
\hline Age (years) & $39.3 \pm 12.7$ & $20-52$ \\
\hline Male gender, n (\%) & $43(100)$ & \\
\hline Weight (kg) & $67 \pm 10.0$ & $57-84$ \\
\hline BMI (kg/m $\left.{ }^{2}\right)^{*}$ & $22.7 \pm 3.8$ & $20-32$ \\
\hline Number of sessions received & 30 & $20-40$ \\
\hline Number of full recovery, $\mathbf{n}(\%)$ & $38(88.37)$ & \\
\hline
\end{tabular}

${ }^{*} \mathrm{BMI}=$ Body Mass Index , ${ }^{*}$ Pearson Chi-Square test for frequencies.

SSNHL patients and laboratory findings.

\begin{tabular}{llll}
\hline Laboratory parameters & Before HBOT & After HBOT & p Values = \\
\hline PON1 (ng/ml) & $19.7 \pm 2.7$ & $17.0 \pm 2.1$ & $0.0036^{*}$ \\
\hline ARE (kU/L) & $228.4 \pm 51.2$ & $178.2 \pm 42.5$ & $0.024^{*}$ \\
\hline TC (mmol/L) & $4.75 \pm 1.2$ & $4.70 \pm 1.1$ & 0.084 \\
\hline LDL (mmol/L) & $2.67 \pm 0.8$ & $2.07 \pm 1.4$ & 0.078 \\
\hline TG/HDL-C & $2.456 \pm 0.87$ & $2.33 \pm 0.93$ & 0.063 \\
\hline HDL (mmol/L) & $1.03 \pm 0.29$ & $1.04 \pm 0.29$ & 0.062 \\
\hline TG (mmol/L) & $2.05 \pm 1.3$ & $2.07 \pm 1.4$ & 0.069 \\
\hline $\begin{array}{l}\text { *Paired samples t-test p<0.05 } \\
\text { Mean } \pm \text { SD=Standard }\end{array}$ & Deviation, & TC=Total cholesterol, $\quad$ HDL-C=High-density $\quad$ lipoprotein-cholesterol, LDL-C=Low-density lipoprotein-cholesterol,
\end{tabular}

TG=Triglycerides.

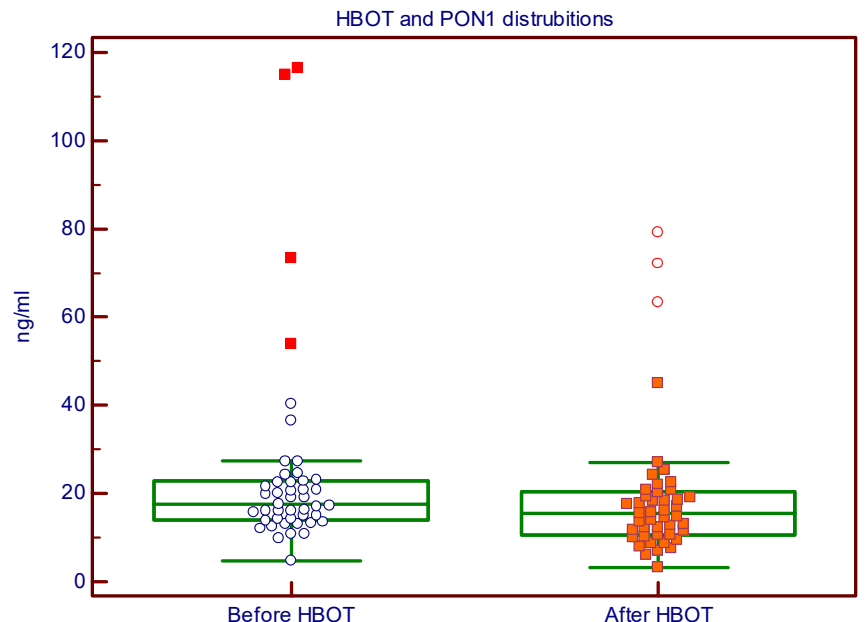

Fig. 1 Multiple comparisons Graph of PON1 amounts before and after HBOT. Distribution of serum PON1 in SSNHL patients. In the Box-and-whisker plot, the central box represents the values from the lower to upper quartile ( 25 to 75 percentile). The middle line represents the median. The horizontal line extends from the minimum to the maximum value, excluding outside and far out values which which are displayed as separate points.

\section{REFERENCES}

1. Eren E, Yilmaz N, Yildirim F, Giray O HDL functionality in patients with hyperbaric oxygen therapy .Internet Journal of Medical Update. 2019 January; 14(1):5-10. doi:10.4314/ijmu.v14i1.2;

2. Paprocki J, Sutkowy P, Piechocki J, Woźniak A.The Oxidant-Antioxidant Equilibrium in the Blood of People with Sudden Sensorineural Hearing Loss After the First Hyperbaric Oxygen Therapy Session - A Preliminary Study PolHypRes .Volume 61: Issue 428 Apr 2018 : 15-24 doi:10.1515/phr-2017-0018;

3. Paprocki J, Sutkowy P, Piechocki J, Woźniak A Markers of Oxidant-Antioxidant Equilibrium in Patients with Sudden Sensorineural Hearing Loss Treated with Hyperbaric Oxygen Therapy.Oxid Med Cell Longev. 2019; 2019: 8472346. doi: 10.1155/2019/8472346;

4. Gul F, Muderris T, Yalciner G, Sevil E, Bercin S, Ergin M, Babademez MA, Kiris M.A comprehensive study of oxidative stress in sudden hearing loss.Eur Arch Otorhinolaryngol. 2017;274(3):1301-1308. doi: 10.1007/s00405-016-4301-1.

5. Uzun G, Mutluoglu M, Metin S.The use of hyperbaric oxygen treatment for sudden sensorineural hearing loss in Europe.Diving Hyperb Med. 2016;46(1):43-6.PMID: 27044462;

6. Berjis N, Moeinimehr M, Hashemi SM, Hashemi SM, Bakhtiari EK, Nasiri S. Endothelial dysfunction in patients with sudden sensorineural hearing loss.Adv Biomed Res. 2016 Jan 27;5:5.doi: 10.4103/2277-9175.174978;

7. Eren E, Yılmaz N, Aydin O, Ellidağ HY. Anticipatory role of high density lipoprotein and endothelial dysfunction: an overview.Open Biochem J.2014:31:8:100-6.doi:10.2174/1874091X01408010100;

8. Eren E, Yildirim F, Giray O Yilmaz N Hyperbaric Oxygen Treatment is Associated with Lipid Inflammatory Response Assessed Using Serum Platelet Activating Factor. PolHypRes 2019,67 2. 103 - 108. doi: 10.2478/phr-2019-0009;

9. Kozakiewicz M, Kedziora-Kornatowska K, Kaczerska D, Siermontowski P, Olszanski R, Krefft K. Influence of exposure in hyperbaric chambers on selected parameters of oxidative stress in professional divers. Undersea Hyperb Med. 2018;45(1):49-54;

10. Dinc ME, Ulusoy S, Is A, Ayan NN, Avincsal MO, Bicer C, Erel O.,Thiol/disulphide homeostasis as a novel indicator of oxidative stress in sudden sensorineural hearing loss.J Laryngol Otol. 2016 May;130(5):447-52. doi: 10.1017/S002221511600092X; 
11. Kaczerska D, Siermontowski P, Kozakiewicz M, Krefft K, Olszański R.Dehydration of a diver during a hyperbaric chamber exposure with oxygen decompression. Undersea Hyperb Med. 2019 Mar-Apr-May;46(2):185-188.

prof dr Necat Yilmaz SBÜ

AEAH Lc-MS/MS laboratory clinical laboratory education director

Antalya, Turkey

e-mail: necatyilmaz@hotmail.com 